\title{
Alla ricerca di vita su Marte: il ruolo della Fisica del Caos
}

\author{
Giorgio Bianciardi, MS, MD, PhD \\ Università di Siena, Dipartimento di Scienze Mediche, Chirurgiche e Neuroscienze, Siena, Italy \\ Tradotto da Nicoló Antonietti
}

Corrispondenza | giorgio.bianciardi@unisi.it

Citation | Bianciardi, Giorgio. 2020. "Alla Ricerca di Vita su Marte: il Ruolo della Fisica del Caos. Tradotto da Nicoló Antonietti” Journal of Big History IV (2): 78-81.

DOI | https://doi.org/10.22339/jbh.v4i2.4241

Riassunto L'analisi caotica/frattale dei dati dell'esperimento LR dei Viking e delle immagini dei Rover Marziani hanno dato nelle nostre mani indizi molto forti della vita presente e passata su Marte, suggerendoci la presenza di microrganismi sul Pianeta Rosso. Ad oggi, la possibile presenza di esseri viventi su Marte è una domanda ancora aperta che non può essere rifiutata.

\section{Una Introduzione Storica alla Fiscia del Caos}

La Fisica (deterministica) che conosciamo dal liceo:

1) è possibile costruire modelli con caratteristiche deterministiche, che ci permettono di prevedere il sistema, verso il futuro, come indietro nel tempo.

2) Il tempo è reversibile.

3) L'oggetto è semplice o riducibile in sistemi semplici. Il nostro Mondo è il regno dell'aumento dell'entropia, la tendenza al disordine.

Nasce un problema, il mondo della biologia:

1) il regno della complessità e dell'ordine

2) della freccia temporale: l'inversione del tempo è impossibile.

3) della teleonomia: il sistema vivente ha finalismo.

Chi ha ragione? La Fisica o la Biologia? Il trionfante determinismo scientifico può essere descritto ricordando le parole di Laplace:

Possiamo considerare lo stato attuale dell'universo come l'effetto del suo passato e la causa del suo futuro. Un intelletto che in un dato momento conosca tutte le forze che animano la natura e le posizioni reciproche dei corpi che lo compongono, se questo intelletto fosse abbastanza vasto da sottoporre i dati all'analisi, potremmo condensare in un'unica formula il movimento dei più grandi corpi dell'universo e quello dell'atomo più leggero; per un tale intelletto nulla potrebbe essere incerto e il futuro come il passato sarebbe presente davanti ai suoi occhi. (Essai philosophique sur les probabilités, 1814, Laplace)

Tutto sembra chiaro. Tutto è compreso. Ma è così?

È interessante notare che ancora oggi impèri tra i Fisici la descrizione Laplaciana del Mondo: "Conoscendo chi sono e come interagiscono fra di loro i quark si può seguire matematicamente l' evoluzione dell'intero Universo. Soprattutto nel passato ma anche nel futuro.» (da: La Nazione, 3 marzo 1995, Giorgio Bellettini, co-scopritore dell'ultimo quark).

Ma, ancora, in Biologia, possiamo oggi leggere: "Il rumore [tipico comportamento indeterministico] permea la biologia a tutti i livelli, dai processi molecolari e subcellulari più elementari alle dinamiche di tessuti, organi, organismi e popolazioni". (Noise in Biology, Rep Prog Phys. 2014 Lev S. Tsimring) Quindi: un universo deterministico come ci dice la Fisica o un universo indeterministico come descrive la Biologia? I primi avvertimenti un secolo fa: il Problema dei 3 Corpi.

Oscar II di Svezia propose un premio: chi era in grado di presentare una soluzione generale che descrivesse il moto di tre corpi in mutua orbita, qualunque fosse la massa e la distanza? Poincaré vinse il premio.. dimostrando che non esisteva alcuna soluzione generale! (Sur 
le problème des trois corps et les equations de la dynamique, H. Poincaré, 1889)

\section{Nasce la Scienza del Caos}

Edward Lorenz era un matematico e meteorologo presso il Massachusetts Institute of Technology. In un giorno di inverno del 1961, Lorenz stava riesaminando una sequenza di dati provenienti da un suo modello. Invece di riavviare l'intera elaborazione, aveva deciso di risparmiare tempo e riavviare l'elaborazione da dove aveva sospeso il calcolo poco prima (pausa caffè). Mentre inizialmente si proponevano gli stessi risultati forniti in una elaborazione precedente, alla fine le serie numeriche iniziavano a divergere, in modo drammatico-dopo poco era persa ogni somiglianza con i calcoli precedenti.

Una lunga storia che permise la nascita di approcci possibili a questo strano mondo indeterministico (il nostro).

Oggi, in Fisica e Biologia, la Fisica del Caos appare chiaramente in grado di descrivere il nostro Mondo reale.

Che cos'è "Caos"? Nel linguaggio quotidiano il "caos" implica l'esistenza di comportamenti imprevedibili. Il Caos, nel senso della Fisica del Caos, incarna principi importanti: in ogni sistema dinamico: estrema sensibilità alle condizioni iniziali dovuta alla non linearità, dinamiche complesse in cui causa ed effetto non sono proporzionali, previsione a lungo termine impossibile, risultando solo possibile una descrizione meramente statistica del sistema stesso.

Gli alberi e gli alberi bronchiali, i tessuti al microscopio, i neuroni, possono essere descritti dalla geometria del Caos (la geometria frattale), con le loro leggi statistiche, come l'auto-somiglianza al cambiamento di scala. Questa nuova geometria ci consente di esaminare e misurar le, entità biologiche, con un significato profondo. La struttura frattale è anche chiaramente visibile nel mondo della Fisica: Sistema Solare, galassie, ...

I Nostri Risultati: Ricerca di Segni di Vita sul Pianteta Rosso per Mezzo dell'Analisi Coatica/Frattale

Analisi Caotica dei Viking LR experiments.

I primi (e unici) esperimenti per la ricerca di vita su Marte furono eseguiti dai Lander Viking nel 1976. Uno di questi, l' l'esperimento di rilascio marcato (LR) di Levin e Straat: iniezioni di composti organici marcati nei campioni di suolo marziano (Figura 1).

Immediatamente dopo le iniezioni di composti organici nei campioni marziani, gas radioattivo si rilasciava dal suolo marziano, avvicinandosi a un plateau. Questi esperimenti vennero condotti molte volte con risultati simili. È interessante notare che la risposta LR a $160^{\circ} \mathrm{C}$ (sterilizzazione)era molto bassa, soddisfacendo così i criteri pre-

missione per la conferma della presenza di vita (Figura 2).

Tuttavia, e fino ad oggi, sorse una controversia nei confronti di una interpretazione biologica dei dati LR: il gascromatografo / spettrometro di massa (GCMS) non aveva trovato alcun composto organico su Marte; nessun composto organico = assenza di vita..oppure il GCMS mancava di forte sensibilità?

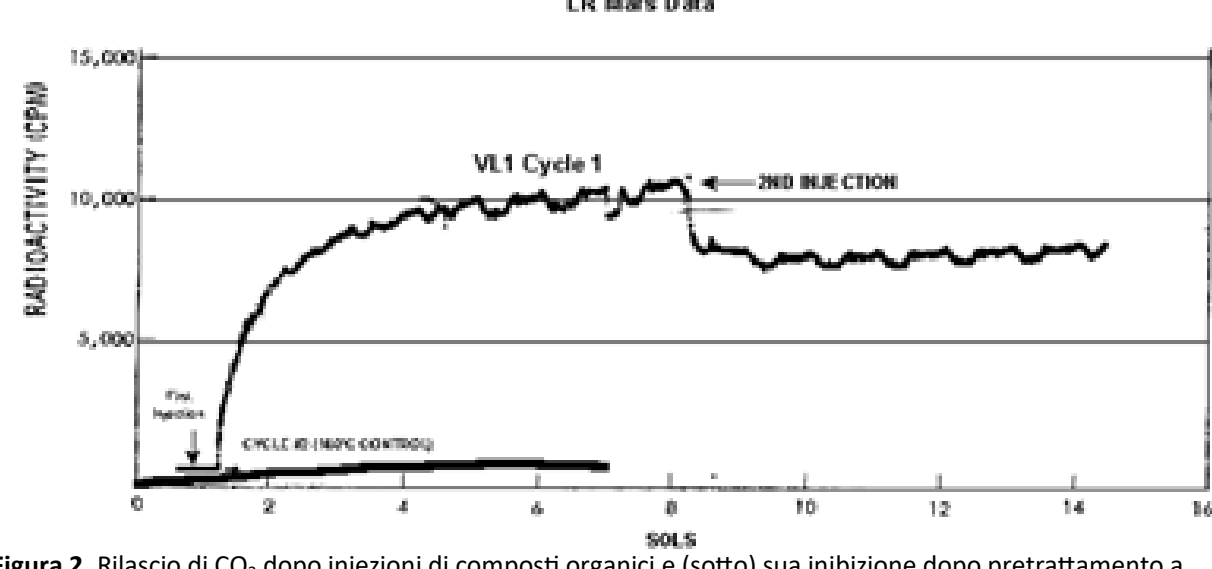

Figura 2. Rilascio di $\mathrm{CO}_{2}$ dopo iniezioni di composti organici e (sotto) sua inibizione dopo pretrattamento a $160^{\circ} \mathrm{C}$. Esperimento LR, Marte.

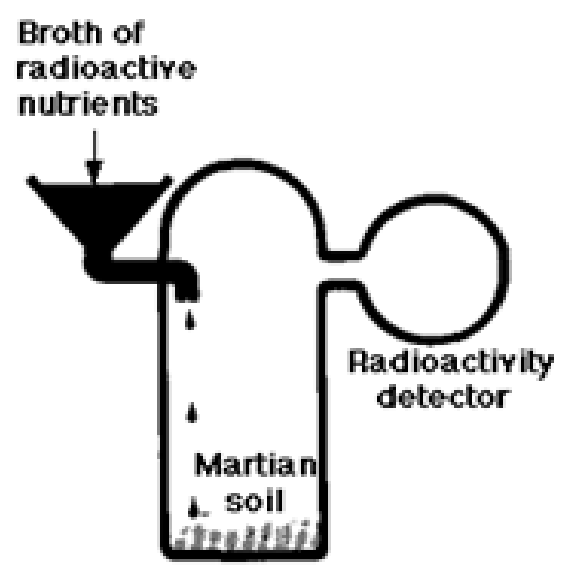

Figura. 1. Labeled Release (LR) experiment

Nel tentativo di risolvere il problema abbiamo impiegato un'analisi caotica dei dati LR marziani e di studi pilota LR terrestri, insieme a dati controllo, biologici e abiologici. In particolare, la rianalisi degli esperimenti di Viking LR è stata da noi eseguita su tutti i 16.000 punti di test sorti dai 9 esperimenti LR effettuati su Marte (attivi, sterilizzati, "affamati", protetti dalla luce solare), nonché dai dati di campioni biologici (controlli positivi, presenza di vita: un test in presenza di batteri terrestri e una serie di 23 giorni di letture della temperatura interna di un ratto) e campioni abiologici (controlli negativi, assenza di vita: radioattività di fondo, misurazioni della temperatura interna del Lander Viking 1, di temperature esterne dell'atmosfera di Marte, oscillazioni della temperatura degli oceani 
sono essere identificate attraverso le

\begin{tabular}{|c|c|c|c|c|c|}
\hline Membe & & & Statistics & & \\
\hline Case & Distance & Variable & Minimum & Mean & Maximum \\
\hline VL2C4 & 0.464 & $\mathrm{LZ}$ & 0.294 & 0.790 & 1.379 \\
\hline Vl1C2 & 0.593 & $\mathrm{H}$ & -0.984 & -0.706 & 0.111 \\
\hline VL1C4 & 0.479 & $\lambda$ & 0.000 & 0.724 & 2.404 \\
\hline VL2C5 & 0.966 & $\mathrm{~K}$ & -1.434 & 0.534 & 1.449 \\
\hline BIOL 6 & 0.311 & BDS & -2.010 & -0.721 & 0.190 \\
\hline DT VL2C3 & 0.790 & $\tau$ & -0.645 & -0.508 & -0.156 \\
\hline VL1 Atmo. temp & 0.413 & & & & \\
\hline Pre-inj radioactivity & 0.494 & & & & \\
\hline
\end{tabular}

Cluster 2 (actives/biological) of 2 Contains 7 Cases

\begin{tabular}{|l|rl|r|r|r|}
\hline \multicolumn{7}{|c}{ Members } & \multicolumn{5}{c}{$\begin{array}{r}\text { Statistics } \\
\text { Minimum }\end{array}$ Mean } \\
\multicolumn{7}{|c|}{ Distance Variable } \\
\hline BIOL5 & 0.534 & LZ & -2.136 & -0.902 & -0.080 \\
VL1C1 & 0.285 & H & -0.218 & 0.806 & 2.190 \\
VL1C3 & 0.409 & $\lambda$ & -1.202 & -0.828 & -0.219 \\
VL2C1 & 0.544 & K & -1.656 & -0.610 & 0.673 \\
VL2C3 & 0.622 & BDS & 0.664 & 0.824 & 1.291 \\
VL2C2 & 0.587 & $\tau$ & -0.174 & 0.581 & 3.304 \\
Rat temp & 1.354 & & & & \\
\hline
\end{tabular}

Tabella 1. Una Cluster analysis separa gli esperimenti LR attivi (Marte e Terra) e i controlli biologici (cluster 2) con i test LR sterilizzati e i controlli abiologici (cluster 1), p <0,001

terrestri). Sette indici non lineari furono utilizzati: Complessità di LempelZiv, Esponenti di Hurst e di Lyapunov, Entropia (Kolmogorov), Correlazione Temporale, BDS (Brock-Dechert-

Scheinkman statistics), Dimensione di Correlazione. L'insieme di tutti i parametri caotici risultò in grado di distinguere gli esperimenti LR attivi su Marte e quelli biologici sulla

Terra dai test di controllo abiotici (p o,oo1) (Tabella 1), permettendoci di sos-

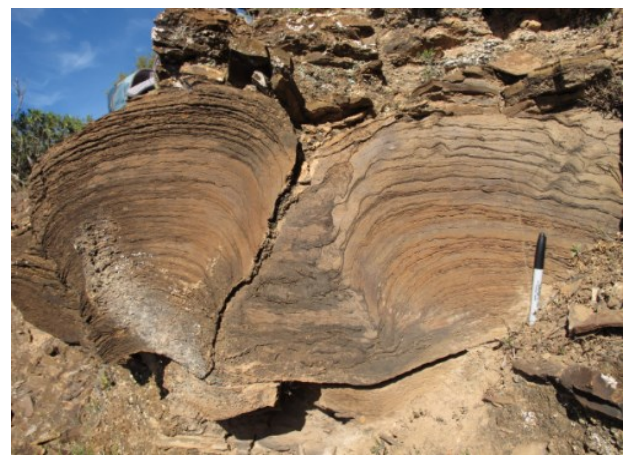

Figura 3. Stromatoliti fossili tenere come gli esperimenti LR avevano davvero rilevato la vita su Marte.

(Bianciardi et al., 2012)

\section{Analisi geometrica frattale degli affioramenti marziani}

Le microbialiti, come le stromatoliti, sono la più antica testimonianza della vita sulla Terra. Le stromatoliti / microbialiti sono un'organizzazione di cianobatteri primitivi in grandi strutture, analoghe alle barriere coralline. Crescono in vaste colonie. Le microbialiti pos-

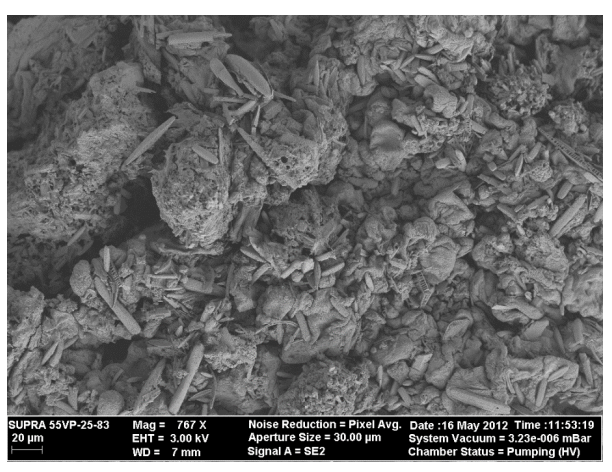

Figura 4. Microsfere e filamenti in una stromatolite vivente ad alto ingrandimento. Microscopia elettronica a scansione, per gentile concessione di M.E. Farias. loro strutture minerali, risultanti dai modelli di crescita dei loro batteri costituenti. Le stromatoliti / microbialiti sono spesso il bersaglio di missioni di rilevamento della vita su Marte (Clarke and Stocke 2013; Jepsen et al., 2007; McKay and Stocke 1989). Ci sono stromatoliti / microbialiti fossili su Marte? Per risolvere il problema, abbiamo eseguito un'analisi frattale delle microstrutture presenti nelle stromatoliti e altri microbialiti sulla Terra, confrontandoli con le microstrutture presenti negli affioramenti fotografati dai rover Opportunity e Spirit rovere. I contorni presenti nelle immagini terrestri e marziane sono stati automaticamente estratti dalle immagini e convertiti in contorni a singolo pixel tramite un filtro Canny. Venne eseguita un'analisi frattale, valutando le immagini terrestri o marziane: complessità geometriche a scala bassa e alta, entropia, a scala bassa e alta, complessità algoritmica (indice Lempel-Ziv o "casualità"), dimensione frattale del minimo percorso (o "tortuosità"), diametro massimo, diametro minimo. L'analisi frattale delle immagini scattate dal rover Opportunity, analizzando 15.000 microstrutture, mostrò parametri frattali che si sovrapponevano a quelli delle microbialiti terrestri: $\mathrm{p}$ $<0,004$ (Tabella 2) (mentre, le pseudostromatoliti abiogeniche presentarono indici morfometrici statisticamente diversi da quelli biogenici) (Bianciardi et al., 2014). Risultati analoghi sono stati otte-

\begin{tabular}{lll}
\hline & $\begin{array}{l}\text { Earth } \\
(\text { mean/SD) }\end{array}$ & $\begin{array}{l}\text { Mars } \\
(\text { mean/SD) }\end{array}$ \\
\hline Complex, High & $1.82(0.02)$ & $1.81(0.02)$ \\
Complex, Low & $1.48(0.05)$ & $1.52(0.07)$ \\
Entropy, High & $1.88(0.01)$ & $1.87(0.02)$ \\
Entropy, Low & $1.41(0.05)$ & $1.44(0.05)$ \\
LZ index & $0.46(0.04)$ & $0.48(0.04)$ \\
Dmin & $0.79(0.03)$ & $0.78(0.03)$ \\
Max Dia (mm) & $0.08(0.001)$ & $0.08(0.001)$ \\
Min Dia $(\mathrm{mm})$ & $0.21(0.003)$ & $0.21(0.003)$
\end{tabular}

Table 2. Fractal parameters and diameters of the Martian microscopic microstructures overlapped the ones of terrestrial biogenic microbialites. The probability of this occurring by chance is less than $p<0.004$. 
nuti analizzando 20.000 microstrutture eseguite dal rover Spirit (Bianciardi et al., 2015).

\section{Riassunto}

Approcci caotici all'esperimento biologico LR eseguito su Marte dai Viking hanno mostrato nelle nostre mani forti evidenze di presenza di vita attuale sul Pianeta Rosso.

L'analisi frattale [la geometria del caos] delle immagini riprese dai rover marziani ha mostrato forti evidenze della presenza di microbialiti su Marte (vita passata sul Pianeta Rosso). Altri investigatori, con approcci diversi, hanno raggiunto conclusioni simili (Noffke, 2015)

Al momento, non esiste alcuna prova definitiva della presenza di vita marziana, ma un numero elevato di evidenze a favore di vita, presente e passata, su Marte sono state raccolte ad oggi. Certamente, la possibile presenza di esseri viventi su Marte è una domanda aperta, ma che non può essere rifiutata.

\section{Bibliografia}

Bianciardi, G. et al. 2015. "Microbialites at Gusev Crater, Mars." Journal of Astrobiology \& Outreach 3, 5.

Bianciardi, G., J. D. Miller, P. A. Straat, and G. V. Levin. 2012. "Complexity Analysis of the Viking Labeled Release Experiments." Journal of Aeronautical and Space Sciences 13 (1): 14.

Bianciardi, G., V. Rizzo, and N. Cantasano. 2014. "Opportunity Rover's Image Analysis: Microbialites on Mars?” International Journal of Aeronautical and Space Sciences 15 (4): 419.

Clarke, J. D., and C. R. Stocker. 2013. "Searching for Stromatolites: The 3.4 ga Strelley Pool Formation (Pilbara Region, Western Australia) as a Mars Analogue." Icarus 224, 413.
Jepsen, S.M., et al. 2007. “The Potential for Lithoautotrophic Life on Mars: Application to Shallow Interfacial Water Environments." Astrobiology 7 (2): 342 .

Laplace, Pierre-Simon. 1840. Essai Philosphique sur les Probabilités. Paris: Bachelier.

Levin, G. V., and P. A. Straat. 1977. "Life on Mars? The Viking Labeled Release Experiment." Biosystem 92, 165.

McKay, C. P. and C. R. Stoker. 1989. "The Early Environment and Its Evolution on Mars: Implications for Life." Reviews of Geophysics 27 (2): 189.

Noffke, N. 2015. "Ancient Sedimentary Structures in the $<3.7 \mathrm{~b}$ Ga Gillespie Lake Member, Mars, That Compare in Macroscopic Morphology, Spatial Associations, and Temporal Succession with Terrestrial Microbialites." Astrobiology 15 (2): 1.

Poincaré, Henri. 1889-189o. "Sur le Problème des Trois Corps et les Equations de la Dynamique." Acta Mathematica 13, 1-270.

Tsimring, Lev S. 2014. "Noise in Biology." Reports on Progress in Physics, Physical Society (Great Britain) 77 (2): 0266o1. doi:10.1088/00344885/77/2/0266o1. 